\title{
Design of New Elderly Care Service Acquisition Mode from the Perspective of Internet Economic Innovation
}

\author{
Yi Ding ${ }^{1, *}$, Zhe Zhang ${ }^{1}$, Meiyun Yang ${ }^{2}$, and Fengqi $\operatorname{Sun}^{1}$ \\ ${ }^{1}$ Nanjing University of Science and Technology, School of Design Art \& Media, Nanjing, Jiangsu, China \\ ${ }^{2}$ Nanjing University of Science and Technology, School of Economics \& Management, Nanjing, Jiangsu, China
}

\begin{abstract}
This research focuses on a service acquisition mode for parents in urban separated families of China to promote the popularization of elderly care services in Internet economy. Based on interviews and questionnaires, authors find the pain point of the elderly care service acquisition mode and propose a tripartite participatory mode. Using this new service acquisition mode, adult children can play an assistant role with a smartphone-based service purchase platform. Next, in order to determine what services should be provided priorly on the platform, an empirical study based on questionnaires and KANO model is carried out to screen a propriate services items. Through the innovation of new elderly care service acquisition mode, more practitioners are supposed to expands their business effectively in aging market, and to play an ever more active role in the growth of Internet economy.
\end{abstract}

\section{Background and purpose}

With the accelerated development of China's aging and urbanization trends in recent years, the aging level of core cities in a region has reduced, while that in surrounding area has enhanced since 2010 [1]. In the meantime, the migration of population to central cities has changed the traditional family structure, posing a great challenge to the traditional elderly care mode [2]. Followed by the increasing pace of life and the reality of separated family members, a new home-based elderly care mode, separated elderly care, is gradually formed [3] and becomes one of the forms of home-based elderly care. The "14th Five-Year Plan" of China proposed to promote the inclusive application of digital services in the elderly care sector. Against the background of booming "Internet+" economy, reconstructing and upgrading the traditional home-based elderly care service mode in combination with Internet is of vital significance for promoting the positive and healthy development of elderly care industry and generating a new economic growth point.

Restricted by the elderly's daily activities, separated elderly care often requires caregivers to provide the elderly with professional elderly care services [4]. As a proven business model, the third-party trading platform based on mobile Internet (hereinafter referred to as "elderly care service platform") not only integrates many commercial resources and trading chains but also is convenient so that it can effectively boost the development of elderly care services. However, at present, such platforms are still relatively scarce. Moreover, considering the popularity of smartphone applications among the elderly population, deepened reflection should be made in the design of services and user experience in order to practically popularize the application of such platform. According to Garrett's theory, elderly care service platform can be divided into strategic layer, scope layer, structure layer, framework layer and performance layer. Service program planning for this platform is in the basic scope layer. As the foundation of detail design, it is a very important step [5]. Although providing plenty service programs can bring more business opportunities, it may increase the burden of users. Hence, it is necessary to maintain a balance between business and user experience by making scientific service plan. In this research, the author conducted empirical research on users by interviews and questionnaire survey and further explored the service acquisition mode and service planning methods in the elderly care service platform based on the result. Through the research, a new practice path is supposed to enlighten practitioners to expand their business in aging market, and more opportunities are incubated to promote the innovation of Internet economic.

\section{Service acquisition mode}

\subsection{Disadvantages of existing mode}

Recent researches in Europe and Asia have revealed that the popularity rate of smartphone in the elderly population and the in-depth use of smartphone are not ideal [6-7]. In this research, in order to verify the availability for the Chinese elderly to use smartphone to obtain elderly care services in the separated elderly care scenario, the author made in-depth and semi-structured

\footnotetext{
$\overline{{ }^{*} \text { Corresponding author: dingyiblue } @ q q . c o m}$
} 
interviews with 21 smartphone users aged 60 who lived in Zhejiang Province and Jiangsu Province, separately with their children, to know about their daily life style, way of acquiring professional service and use of smartphone. The results show that most of the elderly need elderly care service and are willing to receive commercialized door-to-door elderly care service. However, most of them are not proficient in operating smartphone, even they own smartphone. Although they basically master the usage of WeChat, they cannot do more complicated operation, such as placing an order on smartphone to purchase a commodity. With respect to the expected main way of acquiring home-based elderly care services in the future, many of them said that they would ask their children for help or call relevant agency to acquire relevant service.

In home-based elderly care mode, providing professional nursing worker is one of the core elements of such elderly care [8]. Live-in nursing worker can provide the elderly with in-time care and assistance but costs high. In the interview and survey, many old people mentioned that they would feel uneasy if external person participated in their family life over much. Regular doorto-door nursing is an ideal alternative solution but can hardly handle various emergencies in life. Thereby, the elderly care service platform with stronger real-time performance and more optional services will likely become a highly flexible supplementary solution. This platform can not only be used for selecting and ordering nursing workers to provide regular door-to-door elderly care services but also can provide many temporary services when the elderly encounters emergency. Regarding the way of obtaining services, some elderly people said that they can call the service agency as expected. This way is feasible, but the problem is that the integration of service resources is far inferior to that on the elderly care service platform. As discovered in the survey result, a worrying situation is that the usability of smartphone application as a visual carrier of elderly care service platform may be insufficient for the elderly. At present, most of the smartphone applications used to acquire services contain too many function items so the operation method of them is too complex for elders (Fig. 1). Although there are many studies discussing the improvement method for smartphone applications, the biggest limitation of them is the degree of elders' acceptance and mastery of application. Therefore, existing service acquisition mode is intuitive in theory, but it lacks practicality in the current technical conditions.

\subsection{The Role of Adult Children in Service Acquisition Mode}

According to a substantial part of interviewed, in the separated elderly care mode, adult children can assist the elderly acquire elderly care services. Considering the parent-child relationship in the context of Chinese culture, it is highly realistic for many families to have

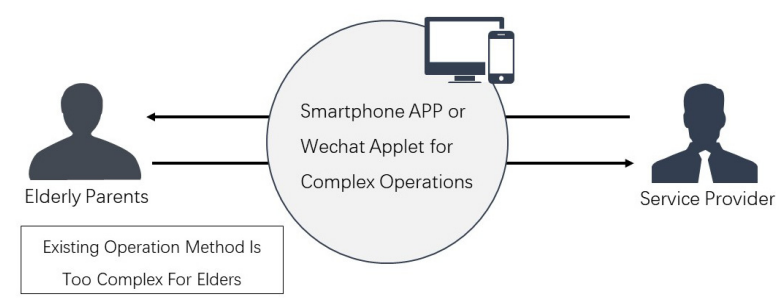

Fig. 1. Existing mode of acquiring elderly care service by smartphone.

adult children play a role in the elderly care process even the elderly and their children live separately. First, the elderly is willing to accept the help of their children to obtain elderly care services. Second, compared with the elderly, their children are more likely to receive elderly care services through smartphone. Further, even the elderly and their children live separately, the way of acquiring service via smartphone-based platform is not restricted in physical distance. Finally, for the elderly contacting with their children frequently, it is less possible to make inappropriate selection due to poor communication. Above all, in China's urban separated families, adult children can not only give important reference opinion to the elderly when selecting services but also can directly help the elderly acquire service in most time. This way can be a beneficial supplement to the home-based elderly care service mode. It is worth noting that in addition to separated adult children, relatives (other than adult children), neighbours, community organizations, etc. can also play similar role under certain circumstances. With the effective participation of adult children, compared with expending a mass of labour and financial costs to the service promotion and communication for the elderly directly, service providers can focus more on the improvement and innovation of service content.

In this paper, a new service acquisition mode is proposed based on the interaction between elderly parents, adult children, and elderly care service provider. In this mode, a third party can access to helping the elderly acquiring elderly care services online on the following premises:

1) Elderly parents at home have the need of obtaining immediate home-based elderly care services, but are somewhat difficult to obtain the services by using smartphone;

2) Parents and children have close interaction, and can communicate the elderly care services at any time;

3) Service providers with professional qualifications put available service items on the elderly care service platform and accept immediate orders.

The core mission of elderly care service platform as a third-party trading platform is to acquire business service. From another point of view, if the platform is treated as a comprehensive solution for separated elderly care, it is also logically and technically possible to get other homebased elderly care related assistant functions incorporated into the platform, such as checking health data, online community, and online consultation, etc. Whereas the screening of various functions relates to 
user experience of the application, user research should be made to screen out and integrate most necessary service items to reduce the over expansion of information and the operational difficulty on the interface.

\section{Service planning for new service acquisition mode}

\subsection{Survey method}

Based on the market survey on some common elderly care service-related websites and smartphone applications in Chinese market, the author selected 23 existing elderly care service platforms, extracted, and classified the service items from them. Finally, 27 common service items were screened out. Those programs not only include the display and screening of elderly care services and the payment related content but also involve some specific services such as information needed for daily life and emergency contact.

In the design of the service platform, in order to clarify that the framework layer needs to select the most important programs from common services to plan the services available on the platform, KANO model (developed by Japanese researcher Noriaki Kano) was used to analyse the relationship between product/service quality and user satisfaction. In this model, service quality is classified into one-dimensional quality (or performance quality), attractive quality, must-be quality, indifferent quality, and reverse quality according to the types of user demands (Fig. 2). Where, one-dimensional quality implies that a certain characteristic of the service is positively correlated to user satisfaction; attractive quality means that certain characteristics can significantly improve user satisfaction, but no dissatisfaction may be caused if lacking such characteristic; indifferent quality indicates that no significant impact may be produced if having or lacking a certain characteristic; and reverse quality represents that certain characteristic is negatively correlated to user satisfaction. KANO model can be used to classify different needs in the questionnaire survey and analyse the action or benefit of specific behaviour in improving the service quality.

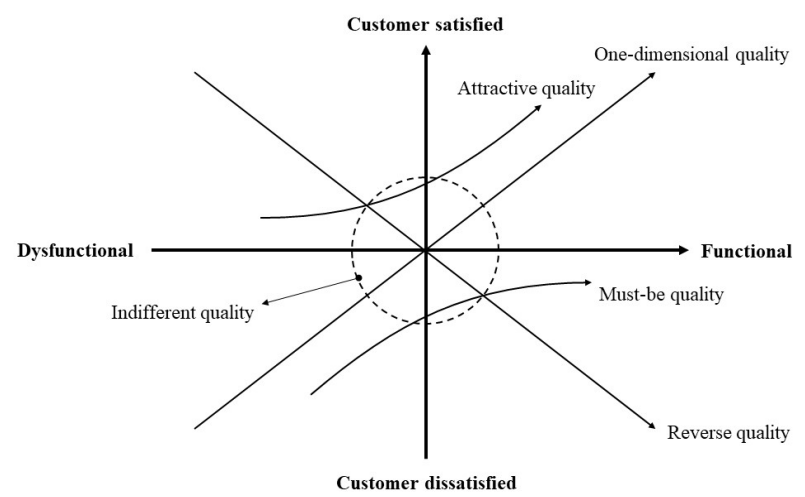

Fig. 2. KANO model.

\subsection{Survey results}

The main target users of the elderly care service platform under the tripartite participatory service mode are smartphone users whose parents have elderly care needs and who are willing to choose and purchase services on behalf of their parents. Hence, the survey objects under this research are the population over 30 years old. They were asked about the said 27 common service items to know their attitudes toward providing and not providing a service and were requested to select an option from the "I like it that way", "It must be that way", "I am neutral", "I can live with it that way" and "I dislike it that way". Eventually, 47 copies of valid questionnaires were collected. After making classified analysis on their answers, the interviewee's attitude toward any service program will lie in a grid of the Kano Evaluation Table (Table 1) which shows the property of the service program. Wherein, A represents attractive quality; M represents must-be quality; $\mathrm{O}$ represents onedimensional; I represent indifferent quality; R represents reverse quality; and $\mathrm{Q}$ represents questionable answers (applicable in case of irrational question setting, or user's misunderstanding or filling error).

Table 1. KANO evaluation table.

\begin{tabular}{|c|c|c|c|c|c|}
\hline & $\begin{array}{c}\text { I like it } \\
\text { that } \\
\text { way }\end{array}$ & $\begin{array}{c}\text { It } \\
\text { must } \\
\text { be } \\
\text { that } \\
\text { way }\end{array}$ & $\begin{array}{c}\text { I am } \\
\text { neutral }\end{array}$ & $\begin{array}{c}\text { I can } \\
\text { live } \\
\text { with it } \\
\text { that } \\
\text { way }\end{array}$ & $\begin{array}{c}\text { I dislike } \\
\text { it that } \\
\text { way }\end{array}$ \\
\hline $\begin{array}{c}\text { I like it } \\
\text { that way }\end{array}$ & Q & A & A & A & O \\
\hline $\begin{array}{c}\text { It must be } \\
\text { that way }\end{array}$ & $\mathrm{R}$ & $\mathrm{I}$ & $\mathrm{I}$ & $\mathrm{I}$ & $\mathrm{M}$ \\
\hline $\begin{array}{c}\text { I am } \\
\text { neutral }\end{array}$ & $\mathrm{R}$ & $\mathrm{I}$ & $\mathrm{I}$ & $\mathrm{I}$ & $\mathrm{M}$ \\
\hline $\begin{array}{c}\text { I can live } \\
\text { with it } \\
\text { that way }\end{array}$ & $\mathrm{R}$ & $\mathrm{I}$ & $\mathrm{I}$ & $\mathrm{I}$ & $\mathrm{M}$ \\
\hline $\begin{array}{c}\text { I dislike it } \\
\text { that way }\end{array}$ & $\mathrm{R}$ & $\mathrm{R}$ & $\mathrm{R}$ & $\mathrm{R}$ & $\mathrm{Q}$ \\
\hline
\end{tabular}

Then, demand satisfaction SI and dissatisfaction DIS were calculated based on the answers. High absolute value of SI indicates that user satisfaction is improved significantly when providing a service; and high absolute value of DIS indicates that user satisfaction is reduced significantly when not providing the service. The calculation equations are as follows, in

$$
\begin{gathered}
\mathrm{SI}=(\mathrm{A}+\mathrm{O}) /(\mathrm{A}+\mathrm{O}+\mathrm{M}+\mathrm{I}) . \\
\mathrm{DSI}=-1^{*}(\mathrm{O}+\mathrm{M}) /(\mathrm{A}+\mathrm{O}+\mathrm{M}+\mathrm{I}) .
\end{gathered}
$$

The concrete calculating methods are as follows: the percentage of the total number of interviewees in each grid in Table 1 is calculated, and brought into (1) and (2) to obtain Si and DSI (Table 2). Next, a four-quadrant scatter diagram of the KANO model was created, taking 
Table 2. Customer Satisfaction Coefficients for Elderly Care Service Platform.

\begin{tabular}{|c|c|c|c|c|c|c|c|c|c|}
\hline Classification of function & $\mathbf{A}$ & $\mathbf{O}$ & $\mathbf{M}$ & $\mathbf{I}$ & $\mathbf{R}$ & $\mathbf{Q}$ & $\mathbf{C a t e g o r y}$ & SI & DSI \\
\hline Introduce service content and flow & 6 & 7 & 4 & 20 & 2 & 8 & $\mathrm{I}$ & $35.14 \%$ & $-29.73 \%$ \\
\hline Screen service personnel & 6 & 10 & 3 & 20 & 0 & 8 & $\mathrm{M}$ & $41.03 \%$ & $-33.33 \%$ \\
\hline View the comments on a specific service & 5 & 9 & 3 & 20 & 0 & 10 & $\mathrm{M}$ & $37.84 \%$ & $-32.43 \%$ \\
\hline View the contact details of offline stores & 3 & 11 & 5 & 21 & 1 & 6 & $\mathrm{M}$ & $35.00 \%$ & $-40.00 \%$ \\
\hline $\begin{array}{c}\text { View the rating rankings of service personnel and } \\
\text { institutions }\end{array}$ & 5 & 10 & 4 & 18 & 0 & 10 & $\mathrm{M}$ & $40.54 \%$ & $-37.84 \%$ \\
\hline Automatically recommend service personnel and institutions & 5 & 8 & 0 & 26 & 1 & 7 & $\mathrm{I}$ & $33.33 \%$ & $-20.51 \%$ \\
\hline View the qualifications of institutions and service personnel & 3 & 11 & 5 & 20 & 0 & 8 & $\mathrm{M}$ & $35.90 \%$ & $-41.03 \%$ \\
\hline View the comments on institutions and service personnel & 4 & 9 & 3 & 23 & 1 & 7 & $\mathrm{I}$ & $33.33 \%$ & $-30.77 \%$ \\
\hline Click "Like" for the service or service personnel you like & 4 & 10 & 1 & 22 & 1 & 9 & $\mathrm{I}$ & $37.84 \%$ & $-29.73 \%$ \\
\hline Online interview service personnel & 8 & 5 & 0 & 25 & 1 & 8 & $\mathrm{I}$ & $34.21 \%$ & $-13.16 \%$ \\
\hline Announce a demand and wait for response online & 6 & 10 & 0 & 21 & 0 & 10 & $\mathrm{I}$ & $43.24 \%$ & $-27.03 \%$ \\
\hline Regularly recommend popular services & 5 & 9 & 1 & 22 & 1 & 9 & $\mathrm{I}$ & $37.84 \%$ & $-27.03 \%$ \\
\hline Top the common or important services & 8 & 8 & 2 & 18 & 0 & 11 & $\mathrm{I}$ & $44.44 \%$ & $-27.78 \%$ \\
\hline Display various services in categories & 7 & 13 & 3 & 13 & 0 & 11 & $\mathrm{O}$ & $58.82 \%$ & $-41.18 \%$ \\
\hline volume & 7 & 9 & 3 & 18 & 1 & 9 & $\mathrm{~A}$ & $45.71 \%$ & $-28.57 \%$ \\
\hline Rank the services according to the ratings or purchasing & & & & & & & & \\
\hline Look over the weather forecast & 7 & 17 & 1 & 10 & 0 & 12 & $\mathrm{O}$ & $70.59 \%$ & $-50.00 \%$ \\
\hline View health-related encyclopedia & 7 & 13 & 1 & 14 & 2 & 10 & $\mathrm{O}$ & $58.82 \%$ & $-38.24 \%$ \\
\hline Online community & 7 & 4 & 3 & 23 & 2 & 8 & $\mathrm{I}$ & $33.33 \%$ & $-15.15 \%$ \\
\hline Paid expedited service & 6 & 7 & 1 & 24 & 2 & 7 & $\mathrm{I}$ & $38.24 \%$ & $-23.53 \%$ \\
\hline View various discount and promotion information & 1 & 4 & 2 & 28 & 3 & 9 & $\mathrm{I}$ & $16.67 \%$ & $-16.67 \%$ \\
\hline Ecan the code of a drug to query the efficacy and expiry date & 13 & 11 & 1 & 17 & 0 & 8 & $\mathrm{~A}$ & $58.33 \%$ & $-30.56 \%$ \\
\hline Check personal health data & 9 & 13 & 2 & 15 & 0 & 8 & $\mathrm{O}$ & $61.11 \%$ & $-36.11 \%$ \\
\hline
\end{tabular}

$\mathrm{SI}$ and DIS as the $\mathrm{Y}$-axis and $\mathrm{X}$-axis respectively and the average of the two as the critical line (Fig. 3).

In the scatter diagram, the first quadrant (onedimensional quality) shows that the satisfaction of interviewees is significantly improved when a specific service is provided, while on the contrary, the satisfaction is significantly reduced; the second quadrant (attractive quality) presents that the satisfaction of interviewees is significantly increased when a specific service is provided, while on the contrary, the satisfaction is not significantly reduced; the third quadrant (indifferent quality) indicates that there is no significant change in the satisfaction and dissatisfaction of interviewees no matter whether a specific service is provided or not; the fourth quadrant (must-be quality) reveals that the satisfaction of interviewees is significantly reduced when a specific service is not provided, while on the contrary, satisfaction is not significantly improved. According to the concept of KANO Model, relevant service items with must-be quality should better be incorporated in the service plan in principle. Taking user satisfaction into consideration, a rational choice in making service plan is to provide many relevant service items with one-dimensional quality. Although relevant service items with attractive quality are not necessary, they can be listed as the key reference factors when facing market competition or wishing to build up own advantages by service design. As for services with indifferent quality, such programs should be carefully screened and then selected as per their specific conditions.

According to the scatter diagram, relevant service items with must-be quality include "Screen service personnel", "View the rating rankings of service personnel and institutions", "View the comments on a specific service", "View the qualifications of institutions and service personnel", "View the contact details of offline stores", relevant service items with onedimensional quality include "Look over the weather forecast", "Pre-set important services and one-touch calling", "Check personal health data", "Alert the guardian when the elderly leaves the pre-set area", "Display various services in categories", "View healthrelated encyclopaedia", relevant service items with attractive quality include "Enter health information for health assessment", "Daily health consultation online", "Scan the code of a drug to query the efficacy and expiry date", "Rank the services according to the ratings or purchasing volume", relevant service items with indifferent quality include "Online community", "Online interview service personnel", "Regularly recommend popular services", "Paid expedited service", etc..

\section{Discussion}

As discovered in the survey, when assisting parents in operating for acquiring elderly care services, adult children think that "View the professional qualifications of institutions and service personnel", "View the contact details of offline stores", "View the comments on institutions and service personnel" and the like service programs should be provided in the elderly care service platform. Enhancing the service programs like "Display various services in categories", "Apply for professional medical service", "Pre-set important services and onetouch calling" and "Alert the guardian when the elderly leaves the pre-set area" contribute to significantly 
Attractive Quality

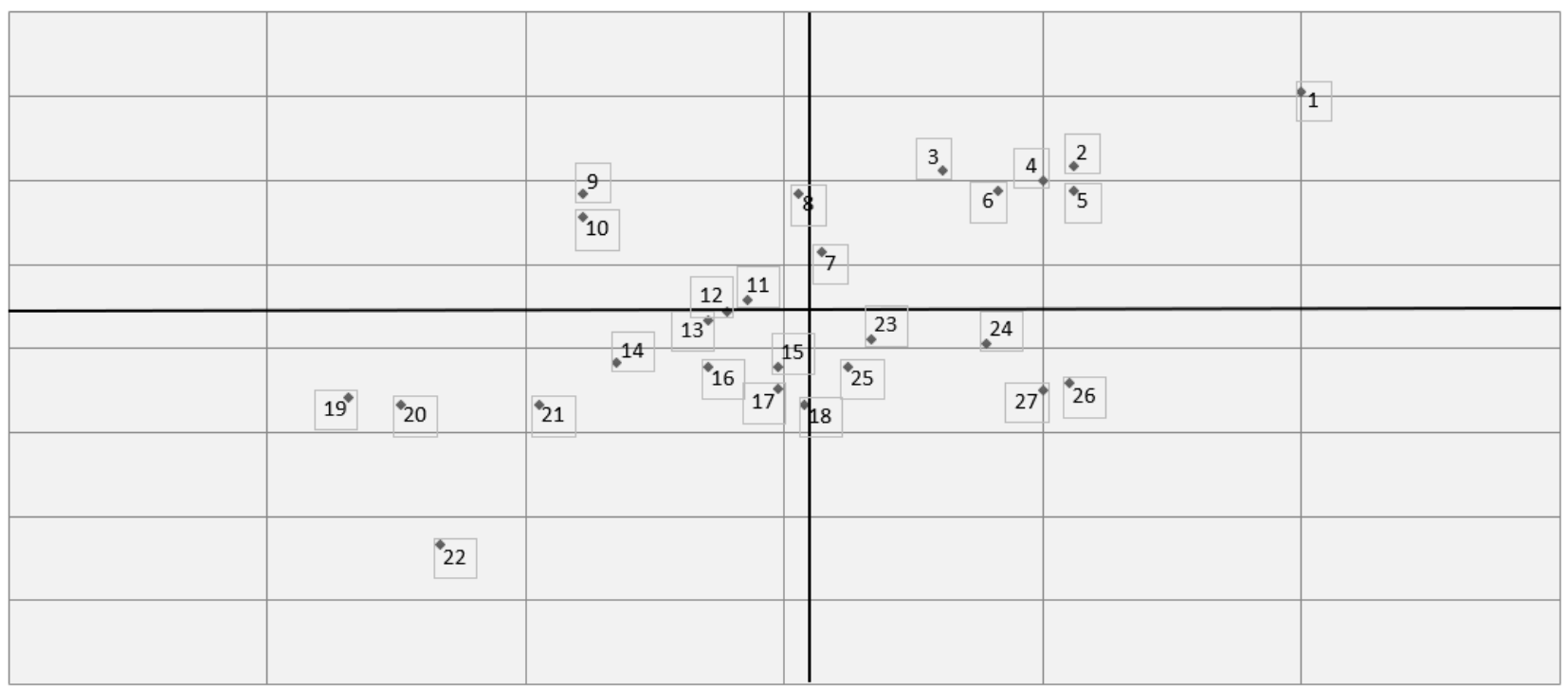

One-dimensional Quality

Must-be Quality

Note: $1=$ Look over the weather forecast; $2=$ Pre-set important services and one-touch calling; $3=$ Check personal health data; 4=Alert the guardian when the elderly leaves the pre-set area; 5=Display various services in categories; 6=View health-related encyclopaedia; 7=Apply for professional medical service; $8=$ Enter health information for health assessment; 9=Daily health consultation online; $10=$ Scan the code of a drug to query the efficacy and expiry date; $11=$ Rank the services according to the ratings or purchasing volume; $12=$ Top the common or important services; 13=Announce a demand and wait for response online; 14=Online community; 15=Click "Like" for the service or service personnel you like; 16=Regularly recommend popular services; 17=Introduce service content and flow; $18=$ View the professional qualifications of institutions and service personnel; $19=$ View various discount and promotion information; $20=$ Online interview service personnel; $21=$ Automatically recommend service personnel and institutions; 22=Paid expedited service; $23=$ Screen service personnel; 24=View the rating rankings of service personnel and institutions; $25=\mathrm{View}$ the comments on a specific service; 26=View the qualifications of institutions and service personnel; $27=$ View the contact details of offline stores.

Fig. 3. Attribute distribution in KANO Model

increase adult children's satisfaction. "Daily health consultation online", "Scan the code of a drug to query the efficacy and expiry date" and "Enter health information for health assessment" are worthy of consideration when expecting to improve the service attractiveness. In addition, "Paid expedited service", "Automatically recommend service personnel and institutions", "Click 'Like' for the service or service personnel you like" and the like service programs are not that greatly correlated to user satisfaction and thus should be carefully selected to ensure the availability of the operation interface of the platform.

The above results reveal that professional property and reliability are the premises that current users consider to select a service. Meanwhile, the learning of professional knowledge, mastering of own health conditions and response to emergency and so on provide clues for making in-depth plan for the service programs. Although the services in the platform have appeared in existing service models, as aforesaid, the third-party trading platform still has the development potential for reason that it integrates a large amount of business resources and trading chains and is specialized in elderly care service. Making clear different types of functional programs is conducive for relevant platform designer to determine the development framework.

Combined the result of filtering service items matching with the premises mentioned in Chapter II of this paper, the new service acquisition mode should be externalized as a tripartite participatory mode, which allows adult children to play a more active role. In this new mode, elderly care service platform is the core for display and transaction of elderly care services. After communication and knowing about parents' demands, adult children can search, screen and place an order of relevant service on the smartphone application (or similar convenient WeChat Applet) of this platform and monitor based on the position, progress and the like information provided by the system. On the other hand, elderly parents do not need to do complicated operation on smartphone application (Fig. 4). Considering the popularity of WeChat in the elderly population, a simplified WeChat Applet can be provided to facilitate them making simple confirmation on the arrival of nursing worker and the completion of service. 


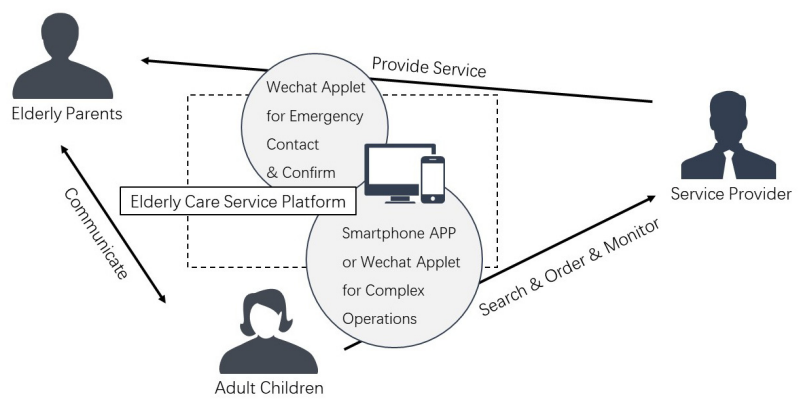

Fig. 4. Tripartite Participatory Service Mode

According to the survey based on Kano model, for adult children, when they use this smartphone application, the priority is to ensure the query and screening of services. Then, some additional services directly related to health is worth considering. However, some of the additional services proposed in this paper are directly aimed at the elderly users, so further studies are needed to find out how to allocate various additional services to the application oriented to adult children and the WeChat Applet oriented to elderly.

In this paper, the authors only discuss the main connotation of the tripartite participatory service mode in Chinese urban separated families. The complex and changeable demands to be met by separated elderly care still need to be further studied. Moreover, busy work, family relations and the like factors may cause absence of the role of adult children in the family, affecting one of the premises set for the tripartite participatory service mode, namely "Parents and children have close interaction, and can communicate the elderly care services at any time". In this case, residential committee, and other grassroots organizations [9], community resident nursing staff [10] and others may all be available to play an alternative role.

Proposing this mode is not only to provide better user experience for the elderly, but also to promote more elderly to accept a third-party trading platform based on smartphone to update the mode of providing elderly care services. In this new elderly care service acquisition mode, service providers can participate in the rapid development of the industry in a more diversified form without being limited by the traditional service types. The beneficiaries of the new mode not only include the elderly and existing practitioners, but also create opportunities for more stakeholders. For example, on the one hand, the new model provides more convenience for adult children to pay attention to their parents' lives and reduce potential safety hazards; on the other hand, it includes many practitioners who were not originally specifically for the elderly, and encourages them to carry out service innovation based on the new platform.

\section{Conclusions}

This research focuses on the elderly care service acquisition mode oriented to Chinese urban separated families, and makes an empirical study by using interviews, questionnaires, and Kano model. According to the results, a tripartite participant service mode is proposed, and the service items it should contain are deeply screened and analysed. In this mode, elderly, adult children, and service providers are connected in a way which considers user experience, safety, and professionalism. With the application of the new mode, the elderly's acceptance of getting pension services through the mobile platform can be expected to rise, which has positive significance for the growth of pension industry in the Internet economy. Therefore, it can be understood as an opportunity to promote the innovation of related industries under the existing technical conditions.

\section{Acknowledgments}

Supported by "the Fundamental Research Funds for the Central Universities”, No. 30920021204.

\section{References}

1. Wang, L. C., Wu, R. W., Li, W. (2016) Spatialtemporal patterns of population aging on China's urban agglomerations. Acta Geographica Sinica, 72(6):1001-1016.

2. Liu, J. Y. (2014) Ageing, migration and familial support in rural China. Geoforum, 51:305-312.

3. Cai, M. H., Wu, G. (2006) The providing mode for the aged in urban separated-living families. Journal of Changsha Social Work College, 13(1):27-29.

4. Dong, H. Y. (2020) Cultures From Filial Piety to Care and Respect for the Elderly__Building a New Cultural System to Adapt to Aging Society. Academic Journal of Zhongzhou, 9: 68-74.

5. Garrett, J. (2011) The elements of user experience. 2nd ed. Berkeley, Calif.: New Riders.

6. Carmien, S., Manzanares, A. G. (2014) Elders using smartphones-a set of research based heuristic guidelines for designers. In: International conference on universal access in human-computer interaction. Cham. pp. 26-37.

7. Wang, L., Dai, J. B. (2020) Investigation and Suggestion on the Use of Smart Phones by the Urban Elderly. Journal of Changsha aeronautical vocational and technical college, 20(4):96-98, 103.

8. Wang, S. M., Zhang, C. N., Chen, Y. Y., Sun, C. X. (2021) Elder-care assistant's attitudes toward government purchases of home-based elderly care services: a qualitative study. Journal of Nursing Science, 36(6): 91-94.

9. Xu, Q.W., Chow, J. C. (2011) Exploring the community-based service delivery model: Elderly care in China. International Social Work, 54(3): 374-387.

10. Yang, H.M., Ye, J. (2015) Application effect of community nursing in health services in home care. China Modern Medicine, 22(25):183-188. 